\title{
Yves-Claude Lequin et Pierre Lamard (dir.), Éléments de démocratie technique
}

Belfort-Montbéliard, université de technologie de Belfort-Montbéliard (Sciences humaines et technologie), 2015

\section{Sezin Topçu}

\section{(2) OpenEdition}

Édition électronique

URL : http://journals.openedition.org/artefact/762

DOI : $10.4000 /$ artefact.762

ISSN : 2606-9245

Éditeur :

Association Artefact. Techniques histoire et sciences humaines, Presses universitaires du Midi

Édition imprimée

Date de publication : 15 juin 2017

Pagination : 255-258

ISBN : 978-2-7535-6525-8

ISSN : 2273-0753

\section{Référence électronique}

Sezin Topçu, «Yves-Claude Lequin et Pierre Lamard (dir.), Éléments de démocratie technique », Artefact [En ligne], 5 | 2016, mis en ligne le 15 novembre 2017, consulté le 23 septembre 2020. URL : http:// journals.openedition.org/artefact/762 ; DOI : https://doi.org/10.4000/artefact.762

Ce document a été généré automatiquement le 23 septembre 2020.

\section{cc) (†)}

Artefact, Techniques, histoire et sciences humaines est mise à disposition selon les termes de la Licence Creative Commons Attribution - Pas d'Utilisation Commerciale - Pas de Modification 4.0 International. 


\title{
Yves-Claude Lequin et Pierre Lamard (dir.), Éléments de démocratie technique
}

\author{
Belfort-Montbéliard, université de technologie de Belfort-Montbéliard
} (Sciences humaines et technologie), 2015

\section{Sezin Topçu}

\section{RÉFÉRENCE}

Yves-Claude Lequin et Pierre Lamard (dir.), Éléments de démocratie technique, BelfortMontbéliard, université de technologie de Belfort-Montbéliard (Sciences humaines et technologie), 2015, $284 \mathrm{p}$.

1 La démocratie technique, notion-modèle qui a eu une carrière brillante à partir des années 1990, a souvent été discutée, tant sur le plan théorique que pratique, à l'aune des technologies controversées et des problèmes de gouvernance qu'elles soulèvent. Elle a en quelque sorte été saisie dans le vif, en vue d'innover un mode de gouvernement opérationnel pour les secteurs techno-industriels émergents ou les produits technoscientifiques contestés. Éléments de démocratie technique, coordonné par Yves-Claude Lequin et Pierre Lamard, déplace la focale pour interroger la réalité et la portée de la démocratie technique (DT) en temps «normal»-et permanentd'activités industrielles, chez, du point de vue, ou en lien avec des acteurs à l'origine de l'offre technologique. L'ouvrage s'intéresse ainsi aux dynamiques participatives dans l'usine ou dans l'entreprise (première partie, sur les espaces d'activités), passe au crible les outils censés œuvrer à la démocratisation de et par la technique (deuxième partie, sur les outils) et donne la parole à des acteurs de terrain pour éclairer la façon dont ils définissent les rapports souvent clivés entre technologie et société et prescrivent des solutions pour y remédier (troisième partie, sur les perspectives); puis il discute les enjeux que représente la formation des ingénieurs dans la fabrique des technologies 
démocratiques ou du moins "pacifiées» (quatrième partie, sur l'enseignement technique). Afin de proposer un tel panorama, les coordinateurs de l'ouvrage ont choisi de réunir historiens, sociologues, philosophes, mais aussi scientifiques, ingénieurs ou encore responsables industriels, dans une sorte de forum non pas contradictoire mais pluriel, pour lequel l'enjeu est moins de définir en amont ce qu'est ou ce que doit être la "démocratie technique " que d'en présenter un état des lieux, des réflexions et des intentions.

2 L'ouvrage s'ouvre par une revue historique des revendications et pratiques démocratiques ou participatives qui ont vu le jour dans le monde du travail. YvesClaude Lequin rappelle l'importance des luttes ouvrières et populaires centenaires (des grèves de Renault de 1913 au mouvement Lip, en passant par le syndicalisme des années 1950) ayant précédé la période soixante-huitarde de luttes autogestionnaires et d'expériences de démocratie directe. L'historien décrit ensuite les réformes institutionnelles associées, dans l'immédiat après Mai 68 (assouplissement du libéralisme autoritaire), puis dans l'après 1981 qui voit le déploiement officiel des dispositifs participatifs destinés dans un premier temps aux salariés, puis aux populations locales (grands projets d'équipement). On ne peut pour autant acter l'avènement progressif d'une ère de démocratie industrielle, tant l'ouverture des possibles pour une réelle émancipation des travailleurs a demeuré et demeure encore aujourd'hui cadrée, conditionnelle, voire illusoire, comme le montre Martine Legris Revel dans son analyse de deux entreprises, française et britannique. Du côté des usagers cependant, soutiennent Léonard Laborie et ses coauteurs, le constat s'avère moins sombre, dans la mesure où une pluralité d'acteurs, dont des associations d'usagers ou de consommateurs, ont historiquement participé à la gouvernance des grands réseaux techniques (téléphone, électricité, chemins de fer), en jetant ainsi, dès la fin du $\mathrm{XIX}^{\mathrm{e}}$ siècle, les jalons d'une démocratie «techno-économique». Une autre approche encore de la démocratisation, ou du moins de la mise aux «normes» de projets techniques, est offerte par Bernard Guy depuis les lieux d'exercice des ingénieurs: il s'agit de la nécessité d'articuler le vrai et le bien, la conception et l'évaluation, le savoir et l'éthique.

3 La deuxième partie du livre organise la discussion autour des outils et innovations techniques, notamment numériques, en faisant se confronter des visions antagonistes. Aussi, Erwin Van Handenhoven, designer au sein d'un grand groupe industriel du secteur du 3D, soutient, par exemple, que l'impression 3D constitue(ra) une révolution pour la démocratisation de la société de consommation, que, dans la ligne de mouvements DIY (Do it yourself) et DIWO (Do it with others), elle permettra aux usagers de non seulement fabriquer eux-mêmes des objets, mais également de procéder à leur réparation rapide, ouvrant ainsi la voie à l'émancipation citoyenne par le "faire ", réinstaurant également les conditions pour un attachement à l'objet, contrepoint de l'obsolescence programmée, garant d'un modèle de production/consommation soutenable, écologique, durable. À l'opposé, Guillaume Carnino dresse une cartographie d'auteurs, de penseurs ou d'activistes (d'Evgeny Morozov à Sherry Turkle, en passant par Jean-Jacques Delfour du côté français, ou encore le groupe Marcuse et le collectif Pièces et Main d'œuvre...), ayant formulé une forte critique vis-à-vis des outils numériques. Loin de renforcer la communication entre individus ou de booster leur productivité (comme dans le cas de 3D...) moyennant des interfaces numériques d'échange et d'apprentissage, Internet et plus généralement le numérique seraient, 
selon ces divers analystes, des outils de déploiement des modes d'être paradoxaux et incompatibles, des outils de "fraude » en quelque sorte, promouvant de l'ignorance dans l'information, de la solitude dans le collectif virtuel, de la présence dans l'absence, de l'impuissance participative, de la démocratie technique sous surveillance autoritaire. Éric Guichard modère ces deux visions polarisées dans son analyse des rapports entre Internet et production de connaissances. Une fois pris acte du fait que le savoir rendu accessible par Internet est trop politique pour permettre la constitution d'une capacité critique ou d'un débat démocratique, le philosophe voit de possibles pistes d'émancipation plutôt du côté de la nouvelle «culture » de l'écrit que propulse Internet, culture ou savoir qui nécessairement doterait les citoyens d'un nouveau type de pouvoir (d'expression et d'agir) selon lui.

4 La troisième partie de l'ouvrage, consacrée aux "perspectives", donne d'abord la parole à un membre de l'Académie des technologies, Bernard Decomps, qui expose sa vision de la DT en phase avec celle livrée par l'Académie dans ses recommandations récentes. Le physicien soutient que la démocratie technique passe par la large diffusion d'une culture technique, tout autant que par la démocratisation de l'accès aux usages (celle d'Internet ou de 3D par exemple). Le cercle élargi d'usagers ou de technologues amateurs serait le garant d'élargissement des cercles décisionnels. On est loin ici des préoccupations relatives à la (nécessaire) mise en évaluation publique et critique des choix technologiques. La technique (e.g. Internet) est plutôt vue comme un support devant faciliter la démocratisation d'autres décisions de la vie publique et non comme un objet de discussion en soi. Le pouvoir (devant être) accordé au citoyen dans les sociétés technologiques est abordé tout à fait différemment par Andrew Feenberg. Le philosophe des techniques canadien, auteur entre autres de Pour une théorie critique des techniques (2014), définit trois conditions pour permettre la capacité d'agir (agency) des citoyens: savoir, pouvoir, opportunité. Selon Feenberg, le réseau technique a fait advenir une nouvelle forme de citoyenneté technologique qui se distingue de grandes interventions (telles que les révolutions) : la micropolitique. Elle limite l'autonomie des experts, permet de nouvelles réglementations, ouvre la voie à de produits plus responsables. Aussi, depuis notre entrée dans l'ère d'Internet, dit-il, les citoyens doivent prendre leur destin en main et intervenir pour identifier les nouvelles trajectoires du progrès, un progrès qui doit se reposer sur une conception différente de la richesse, de l'abondance, de la modernité. De manière convergente, Victor Petit propose une nouvelle approche de la technocratie (et de sa critique) pour rendre possible le mariage entre technique et démocratie. Selon le philosophe, une fois pris acte du caractère politique des techniques, constat désormais banal, il faut subvertir la perspective analytique en appréhendant la «culture technique » au sens de Simondon (c'est-à-dire une culture technique qui sait combien chaque artefact ou chaque algorithme fait de « la politique »), en formant des «ingénieurs démocrates » dotés de cette culture, en promouvant ainsi l'avènement des technologies démocratiques (au sens de Lewis Mumford, Langdon Winner, mais aussi Tim Mitchell et Richard Sclove) plutôt que d'essayer de faire advenir la démocratie technique, un objectif trop peu ciblé qui selon l'auteur dépolitise les choix techniques.

5 Enfin, la dernière partie de l'ouvrage réfléchit à la manière dont l'enseignement technologique peut et doit servir de tremplin pour cultiver une réflexivité démocratique/participative à la source, c'est-à-dire lors de la fabrique des ingénieurs eux-mêmes. Pierre Lamard retrace les avancées relatives à l'insertion des SHS dans la formation des ingénieurs depuis les années 1960 et plus fortement depuis 1970-1980, en 
tant qu' « appareil critique ». Il constate que les SHS souffrent, encore aujourd'hui, d'un statut de supplément d'âme, dans les écoles d'ingénieurs ou les facultés des sciences. Selon lui, le modèle d'« université de technologie » qui occupe une position historique dans la place assez importante octroyée aux SHS dans la formation de l'ingénieur, serait un exemple à suivre. Mais, au-delà d'une délégation aux SHS de la conversion démocratique des ingénieurs-technologues, les modalités de l'enseignement technique lui-même peuvent être avantageusement revisitées. Les contributions de John Didier, puis de Marianne Chouteau et ses collègues explorent cette piste, en partant de l'exemple des dispositifs pédagogiques mis en place en Suisse romande, il y a quelques années, dispositifs centrés sur des activités créatrices et manuelles et attentifs aux phases de socialisation de l'objet technique (fonction, usage, devenir...), et de l'exemple d'un module d'enseignement interdisciplinaire de l'Insa-Lyon, destiné à faire entrer dans la culture technique des ingénieurs les notions de co-conception et de coconstruction de la technique. Toutefois, malgré l'utilité indéniable des innovations pédagogiques visant à sensibiliser l'ingénieur à la "carrière " sociale de l'objet technique qu'il conçoit ou fabrique, il faut aussi remarquer que son activité s'insère dans des contextes économiques et politiques plus vastes et doit nécessairement répondre à (des exigences) des politiques scientifiques et technologiques, élaborées par des instances gouvernementales, nationales, voire supranationales. De fait, la formation des ingénieurs ou de (futurs) cadres techniciens aux enjeux de l'expertise scientifique, aux politique(s) de la science ou aux relations entre science et politique, s'avère tout aussi cruciale, une piste fortement investie au Conservatoire des arts et métiers à partir des années 1980 et dont Vincent Dray retrace ici l'historique.

6 À travers cette riche palette d'entrées, de démonstrations et de débats actualisés, Éléments de démocratie technique enrichit les pistes pour répondre à un besoin méthodologique majeur, celui qui consiste à observer de façon située les différents conceptions, appréciations et retours d'expérience relatifs à la "démocratie technique " plusieurs décennies après les premières théorisations. Au fond, si on prend acte $d u$ fait que les modes d'être ou d'agir démocratiques, mais aussi les formes technocratiques ou les modes de vie technologiques sont, dans nos sociétés contemporaines, en constante transformation, les voies analytiques engagées pour les décrire sont nécessairement variables, voire éclatées, mais elles sont tout aussi inépuisables.

\section{AUTEURS}

SEZIN TOPÇU

CNRS-CEMS-IMM 